Our Nature (2010) 8

\title{
References
}

Bordoloi, R.P.M. 1974. Studies on algal flora of Assam, Gauhati University, Guwahati. (Ph.D. Thesis)

Bruhl, P. and K. Biswas 1922. The algae of Bengal filter beds. J. Deptt. Sci. Calcutta University. 4: 6-10.

Deka, M. and R.P.M. Bordoloi 1991. Studies on bluegreen algae from rice fields of Assam, A qualitative assessment. Phykos 30: 173-180.

Desikachary, T.V. 1959. Cyanophyta. Indian Council of Agricultural Research, New Delhi.

Fogg, G.E. 1949. Culture media for algae. Appendix III. In The cultivation of algae (Ed. G.S. Venkataraman). ICAR, New Delhi. pp. 237246.

Fritsch, F.E., 1945. The structure and reproduction of the Algae. Vol. 2, Cambridge. 939 p.

Geitler, L. 1932. Cyanophyceae in Rabenhorst's Kryptogamenflora. Leipzig 14: 1196.

Hazarika, D. 1988. Distribution of blue green algae of rice-field of Golaghat sub-division (now district), Assam. Gauhati University, Guwahati. (Ph.D. Thesis).

Parukutty, P.R. 1939. On a collection of algae from Assam. Proc. Ind. Acad. Sci. IX. B.

Prescott, G.W. 1984. The algae: A review. Otto Koeltz Science Publisher, West Germany. pp. 141-153.

Saikia, P. and R.P.M. Bordoloi 1994. Blue -green algae of the rice fields of Barpeta, Nalbari and Kamrup district of Assam. Phykos 33(1-2): 53-58.

\section{Fern and Fern-Allies of Eastern Terai, Nepal}

\author{
I.M. Bhagat ${ }^{1 *}$ and S. Shrestha ${ }^{2}$ \\ ${ }^{1}$ Department of Botany, Post Graduate Campus, Tribhuvan University, Biratnagar, Nepal \\ ${ }^{2}$ Department of Botany, Padma Kanya Campus, Baghbazaar, Kathmandu, Nepal \\ *E-mail: indramani@ntc.net.np
}

Received: 18.10.2010, Accepted: 29.11.2010

Key words: Fern, fern allies, enumeration, Polypodiaceae, Nepal.

The eastern Terai covers 620369 hectare area in the southern part of Mechi, Koshi and Sagarmatha zone which comprises 5 districts viz. Jhapa, Sunsari, Morang, Saptari and Siraha. The soil is alluvial, dark grayish to brown in colour with sandy loam to sandy silt in texture. The climate is tropical and sub-tropical and vegetation is predominated by broad leaved, wet monsoonic deciduous forest.

Nepal consists of more than 500 species of ferns and fern-allies. Ferns are generally known as "Unyu/Oony" in Nepali. There are some medicinal ferns, which are regularly exploited both for ayurvedic formulations and traditional healing 
practices. The different species of ferns are also used as vegetables in rural areas in Nepal.

Literatures on the Nepalese Pteridophytes are not sufficient, except some casual references have been made by earlier workers (Raizada and Vaid, 1952; Tagawa, 1975; Panday, 1962; Shakya, 1965; Iwatsuki, 1988; Shrestha and Gurung, 1985; Gurung, 1991; Subba et al., 2001; Thapa, 2002).

The present work is based on one year 2001-2002 of intensive and extensive study of ferns of eastern terai, Nepal, in the field, library, laboratory and herbarium. The ferns were collected from different places of five districts. During the exploration, care was taken to visit all the areas located in different districts to collect plants in fruiting stage. Plant materials and field data were carefully gathered. The identification of collected materials was confirmed by cross checking with the authentic specimens at Tribhuvan University Herbarium, P.G. campus, Biratnagar and National Herbarium and Plant Laboratories, Kathmandu. The cited specimens are preserved in Department of Botany, Post graduate campus, Biratnagar.

The present record includes 35 species of ferns and fern allies belonging to 28 genera and 23 families (Tab.1). Out of these the dominant families are Polypodiaceae (5), Adiantaceae (4), Pteridaceae (3). Three families Schizaeaceae, Thelypteridaceae, Dryopteridaceae are represented by 2 species.
Seventeen families are represented by single species. The members of Athyriaceae, Dryopteridaceae, Pteridaceae and Thelypteridaceae are dominant terrestrial species where as the Marsilea sp., Azolla sp. and Ceratopteris sp., are aquatic, similarly the members of Nephrolepidaceae, Polypodiaceae and Vittariaceae are epiphyte and the members of Adiantaceae, Sinopteridaceae are lithophyte, $2 \mathrm{sp}$. of Schizaeaceae (Lygodium sp.) are climbing ferns and Cyathea spinulosa is an interesting tree fern.

The dominant genera are Adiantum (4) and Pteris (3). Ferns used as green vegetables are Diplazium esculentum, Dryopteris cochleata, Ophioglossum sp. and Tectaria coadunate. Similarly ferns with medicinal values are Adiantum capillusveneris (plants in bronchial and throat pain), A. incisum (fronds in cough fever and diabetes), A. philippense (leaves, rhizome and spores in dysentery and diarrhoea), Diplazium esculentum (rhizome in tuberculosis), Lygodium flexuosom (rizome and leaves as expectorant) and Tectaria coadunate (fronds and rhizome in diarrhoea and dysentery). Ferns with ornamental values are Adiantum capillus-veneris, Cyathea spinulosa, Phymatosorus cuspidatus, Nephrolepsis cordifolia etc.

\section{Acknowledgements}

Grateful thanks are due to Prof. S.D. Joshi, Central Department of Botany, Tribhuvan University and Mr. N. Thapa, National Herbarium and Plant Laboratories, 
Our Nature (2010) 8

Table 1. List of fern and fern-allies of East Nepal

\begin{tabular}{|c|c|c|}
\hline Family & Scientific name & Locality \\
\hline Lycopodiaceae & Lycopodium cernum $\mathrm{L}$. & $\begin{array}{l}\text { Terrestrial, frequent on forests edges at base camp, } \\
\text { Dharan, July-Aug., Bhagat \& Shrestha } 1\end{array}$ \\
\hline Selaginellaceae & Selaginella ciliaris (Retz.) Spring & $\begin{array}{l}\text { Terrestrial, frequent on sandy slopes at Char koshe } \\
\text { jungle, Aug.-Sep., Bhagat \& Shrestha } 2\end{array}$ \\
\hline Equisetaceae & $\begin{array}{l}\text { Equisetum debile Roxb. ex } \\
\text { Vaucher }\end{array}$ & $\begin{array}{l}\text { Terrestrial, abundant along roadsides at Biratnagar, Sep.- } \\
\text { Oct., Bhagat \& Shrestha } 3\end{array}$ \\
\hline Ophioglossaceae & Ophioglossum reticulatum $\mathrm{L}$. & $\begin{array}{l}\text { Terrestrial, common forest floor at Charkoshe, Aug.- } \\
\text { Sep., Bhagat \& Shrestha } 4\end{array}$ \\
\hline Gleicheniaceae & $\begin{array}{l}\text { Gleichenia gigantea Wall. ex } \\
\text { Hook. }\end{array}$ & $\begin{array}{l}\text { Terrestrial, frequant along at Sanischare Jhapa, Jul.-Aug., } \\
\text { Bhagat \& Shrestha } 5\end{array}$ \\
\hline \multirow[t]{5}{*}{ Polypodiaceae } & Drynaria quercifolia (L.) J. Sm. & $\begin{array}{l}\text { Epiphitic, frequent on tree trunks in forest at Charkoshe, } \\
\text { Aug.-Oct., Bhagat \& Shrestha } 6\end{array}$ \\
\hline & $\begin{array}{l}\text { Lepisorus loriformis (Wall. ex } \\
\text { Mett) Ching. }\end{array}$ & $\begin{array}{l}\text { Epiphytic, occassional on tree trunk in forest at } \\
\text { Charkoshe Jhadi, Jun.-Aug., Bhagat \& Shrestha } 7\end{array}$ \\
\hline & $\begin{array}{l}\text { Microsorium embranaceum (D. } \\
\text { Don) Ching. }\end{array}$ & $\begin{array}{l}\text { Epiphytic, frequent on tree trunk in forest at Tarahara, } \\
\text { Jul.-Sep., Bhagat \& Shrestha } 8\end{array}$ \\
\hline & $\begin{array}{l}\text { Phymatosorus cuspidatus (D. } \\
\text { Don) Pich. Serm. }\end{array}$ & $\begin{array}{l}\text { Terrestrial, common on moist \& sunny places of } \\
\text { Charkoshe forest, Jul.-Sep., Bhagat \& Shrestha } 9\end{array}$ \\
\hline & $\begin{array}{l}\text { Pyrrosia porosa }(\text { C. Prest }) \\
\text { Hovenkamp }\end{array}$ & $\begin{array}{l}\text { Epiphytic, common on shady parts of Charkoshe Jungle, } \\
\text { Jul.-Sep., Bhagat \& Shrestha } 10\end{array}$ \\
\hline \multirow[t]{2}{*}{ Schizaeaceae } & Lygodium flexuosum (L.) Sw. & $\begin{array}{l}\text { Climber, frequnt at Charkoshe Jungle, Aug.-Oct., Bhagat } \\
\& \text { Shrestha } 11\end{array}$ \\
\hline & $\begin{array}{l}\text { Lygodium japonicum (Thunb.) } \\
\text { Sw. }\end{array}$ & $\begin{array}{l}\text { Climber, common in moist \& exposed areas at } \\
\text { Charkoshe, Sep.-Nov., Bhagat \& Shrestha } 12\end{array}$ \\
\hline Parkeriaceae & $\begin{array}{l}\text { Ceratopteris thalictroides (L.) } \\
\text { Brong. }\end{array}$ & $\begin{array}{l}\text { Aquatic, common in marshy places at Biratnagar, Jun.- } \\
\text { Aug., Bhagat \& Shrestha } 13\end{array}$ \\
\hline \multirow[t]{4}{*}{ Adiantaceae } & $\begin{array}{l}\text { Adiantum capillus-veneris (L.) } \\
\text { Sp. }\end{array}$ & $\begin{array}{l}\text { Terrestrial, common in rocky places at Baraha Chhetra, } \\
\text { Jul.-Sep., Bhagat \& Shrestha } 14\end{array}$ \\
\hline & Adiantum incisum Forssk. & $\begin{array}{l}\text { Terrestrial, common in rock crevices, slopes and walls at } \\
\text { Charkoshe, Jul.-Sep., Bhagat \& Shrestha } 15\end{array}$ \\
\hline & $\begin{array}{l}\text { Adiantum caudatum (L.) Mant } \\
\mathrm{Pl} \text {., }\end{array}$ & $\begin{array}{l}\text { Terrestrial, muddy rocks at Dharan, Jul.-Sep., Bhagat \& } \\
\text { Shrestha } 1\end{array}$ \\
\hline & Adiantum philippense (L.) Sp. & $\begin{array}{l}\text { Terrestrial, common in rock crevices, slopes and walls at } \\
\text { Charkoshe, Jul.-Sep., Bhagat \& Shrestha } 17\end{array}$ \\
\hline Cryptogrammaceae & Onychium siliculosum (Desv.) C. & $\begin{array}{l}\text { Terrestrial, common on exposed sandy slopes of forests } \\
\text { at Panchkanya, Aug.-Oct., Bhagat \& Shrestha } 18\end{array}$ \\
\hline Hemionitidaceae & $\begin{array}{l}\text { Pityrogramma calomelanos (L.) } \\
\text { Link. }\end{array}$ & $\begin{array}{l}\text { Terrestrial, common in rocky slopes and stream banks of } \\
\text { shady areas at Dharan, Aug.-Oct., Bhagat \& Shrestha } 19\end{array}$ \\
\hline \multirow[t]{3}{*}{ Pteridaceae } & Pteris biaurita $\mathrm{L}$. & $\begin{array}{l}\text { Terrestrial, common in moist places at Charkoshe, Aug.- } \\
\text { Oct., Bhagat \& Shrestha } 20\end{array}$ \\
\hline & Pteris pellucida C. Persl. & $\begin{array}{l}\text { Terrestrial, scattered in moist shady areas at Dharan, } \\
\text { Aug.-Oct., Bhagat \& Shrestha } 21\end{array}$ \\
\hline & Pteris vittata $\mathrm{L}$. & $\begin{array}{l}\text { Terrestrial, common on roadside drains \& walls at } \\
\text { Biratnagar, Jul.-Sep., Bhagat \& Shrestha } 22\end{array}$ \\
\hline Sinopteridaceae & $\begin{array}{l}\text { Cheilanthes bicolar (Roxb.) } \\
\text { Fraser-Jenkins }\end{array}$ & $\begin{array}{l}\text { Terrestrial, common on exposed rock crevice at } \\
\text { Panchkanya, Aug.-Sep., Bhagat \& Shrestha } 23\end{array}$ \\
\hline Vittariaceae & Vittaria sp. J. Sm. & Epiphitic, common in damp tropical at Dharan, Jul.-Sep., \\
\hline
\end{tabular}


Our Nature (2010) 8

\begin{tabular}{|c|c|c|}
\hline & & Bhagat \& Shrestha 24 \\
\hline Marsileaceae & Marsilea minuta L. & $\begin{array}{l}\text { Aquatic, common on the stream sides \& marshes at } \\
\text { Biratnagar, Jul.-Aug., Bhagat \& Shrestha } 25\end{array}$ \\
\hline Cyatheae & Cyathea spinulosa Wall. ex Hook & $\begin{array}{l}\text { Terrestrial, common on the stream at Biratnagar, Jul.- } \\
\text { Aug., Bhagat \& Shrestha } 26\end{array}$ \\
\hline \multirow[t]{2}{*}{ Thelypteridaceae } & Thelypteris prolifera C. Reed & $\begin{array}{l}\text { Terrestrial, common near water sources \& garden at } \\
\text { Biratnagar, Aug.-Oct., Bhagat \& Shrestha } 27\end{array}$ \\
\hline & $\begin{array}{l}\text { Thelypteris dentata (Forssk.) St. } \\
\text { John }\end{array}$ & $\begin{array}{l}\text { Terrestrial, common in open moist places along } \\
\text { roadsides at Dharan, Aug.-Oct., Bhagat \& Shrestha } 28\end{array}$ \\
\hline Athyriaceae & \multicolumn{2}{|c|}{$\begin{array}{l}\text { Diplazium esculentum (Retz.) Sw.Terrestrial, common in moist and rocky places at } \\
\text { Sitaganj, Aug.-Oct., Bhagat \& Shrestha } 29\end{array}$} \\
\hline \multirow[t]{2}{*}{ Dryopteriadaceae } & $\begin{array}{l}\text { Dryopteris cochleata (D. Don) C } \\
\text { Chr. }\end{array}$ & $\begin{array}{l}\text { Terrestrial, scattered in shady slopes at Panchakanya, } \\
\text { Aug.-Oct., Bhagat \& Shrestha } 30\end{array}$ \\
\hline & $\begin{array}{l}\text { Polystichum squarrosum (D, } \\
\text { Don) Fee. }\end{array}$ & $\begin{array}{l}\text { Terrestrial, common in damp places at Charkoshe forests } \\
\text { Jul.-Aug., Bhagat \& Shrestha } 31\end{array}$ \\
\hline Tectariaceae & $\begin{array}{l}\text { Tectaria coadunate (J. Sm.) C. } \\
\text { Chr. }\end{array}$ & $\begin{array}{l}\text { Terrestrial, common in moist places and rocky slopes, } \\
\text { Aug.-Oct., Bhagat \& Shrestha } 32\end{array}$ \\
\hline Nephrolepidaceae & Nephrolepi cordifolia (L.) Presl. & $\begin{array}{l}\text { Terrestrial, common in moist places and along open } \\
\text { slopes of roadsides, also cultivated in gardens, Dharan, } \\
\text { Jul.-Sep., Bhagat \& Shrestha } 33\end{array}$ \\
\hline Blechnaceae & Blechnum orientale $(\mathrm{L})$. & $\begin{array}{l}\text { Terrestrial, occasional on slopes of forest edges at Pathar } \\
\text { \& Jhapa, Jul.-Sep., Bhagat \& Shrestha } 34\end{array}$ \\
\hline Azollaceae & Azolla imbricata (Roxb.) & $\begin{array}{l}\text { Floating aquatic, common in ditches, rice fields, ponds, } \\
\text { Tarahara, Jul.-Sep., Bhagat \& Shrestha } 35\end{array}$ \\
\hline
\end{tabular}

Kathmandu for plant identification. We also thank to U.G.C., Nepal for the financial support.

\section{References}

Gurung, V.L. 1991. Ferns the beauty of Nepalese flora. Sahayogi Press Pvt. Ltd., Kathmandu.

Iwatsuki, K. 1988. An enumeration of the pteridophytes of Nepal. In The Himalaya plants (Eds. H. Ohba and S.B. Malla), Univ. Tokyo Bull. 31: 231-339.

Panday, B.D. 1962. Some aspects of the vegetation of Nepal. Bull. Bot. Survey India 4(4): 135-140.

Raizada, M.B. and K.M. Vaid 1952. Fern of Nepal. India Forester 78: 576-581.

Shakya, A.R. 1965. Cytotaxonomycal studies on ferns of the Kathmandu valley and its environs. Patana University, Patna. (Ph.D. thesis)
Shrestha, R. and V.L. Gurung 1985. A study on ecology of eusporangiate ferns of Nepal Himalaya. In Proceedings of National Conference on Science and Technology, Apr. 24-27, 1988. Royal Nepal Acadamy of Science and Technology, Kathmandu. pp. 359-364.

Subba, D.K., B.K. Rai and M.R. Dhakal 2001. Food value of some edible ferns from Dharan, south eastern Nepal. J. Bomb. Nat. Hist. Soc. 98(3): 499-502.

Tagawa, M. 1975. Contribution to the ferns of Annapurna-Dhaulagiri range, central Nepal. J. Bomb. Nat. Hist. Soc. 72(3): 728-731.

Thapa, N. 2002. Pteridophytes of Nepal. National Herbarium and Plant Laboratories, Department of Plant Resources, Ministry of Forests and Soil Conservation, His Majesty's Government (Nepal), Lalitpur. 175 p. 